\title{
Cuidados paliativos e Alzheimer: concepções de neurologistas
}

\author{
Palliative care and Alzheimer: neurologists' conceptions \\ Cuidados paliativos y Alzheimer: concepciones de neurólogos
}

\author{
Ronaldo Bezerra de Queiroz'; Ana Aline Lacet Zaccara ${ }^{\text {II }}$; Márcia Adriana Dias Meirelles Moreiralli; \\ Luípa Michele Silva ${ }^{I V}$; Solange Fátima Geraldo da Costav; Antonia Oliveira Silva ${ }^{\mathrm{VI}}$
}

\begin{abstract}
RESUMO: O presente estudo teve o objetivo de conhecer concepções de médicos neurologistas acerca da prática dos cuidados paliativos direcionada para o doente de Alzheimer. Trata-se de um estudo exploratório de abordagem qualitativa, desenvolvido em clínicas e hospitais localizados em uma capital do Nordeste brasileiro com 10 médicos neurologistas, que trabalham com portadores de Doença de Alzheimer. A coleta de dados foi realizada no período de abril e maio de 2012. Para a apreensão do material empírico, utilizou-se um formulário, em entrevistas. O material foi tratado por meio da técnica de análise de conteúdo temática categorial. Da análise qualitativa emergiram três categorias: finalidades dos cuidados paliativos; tipos de cuidados paliativos; e formas de enfrentamento. Os depoimentos dos médicos participantes da investigação deixaram transparecer de modo enfático a relevância dos cuidados paliativos para o doente de Alzheimer e sua família. Ante o exposto, pretende-se que esta pesquisa possa subsidiar novos estudos na referida área.

Palavras-Chave: Cuidados paliativos; Doença de Alzheimer; medicina; envelhecimento.
\end{abstract}

ABSTRACT: This study aimed to discover neurologists views on the practice of palliative care for Alzheimer's patients. This qualitative, exploratory study was conducted at clinics and hospitals located in a state capital in northeast Brazil. Empirical data was collected using a form, in the period of April and May 2012, in interviews of 10 neurologists who treat patients with Alzheimer's disease. The material was treated by Theme/Category-Based Content Analysis. The qualitative analysis revealed three categories: goals of palliative care; palliative care; and ways of coping. The declarations of the participating physicians revealed emphatically the importance of palliative care to Alzheimer's patients and their families. In that light, it is intended that this research will support further research in this area.

Keywords: Palliative care; Alzheimer disease; medicine; aging.

RESUMEN: Este estudio tuvo como objetivo comprender los conceptos de los neurólogos sobre la práctica de los cuidados paliativos dirigidos a los pacientes de Alzheimer. Este es un estudio exploratorio de abordaje cualitativo, desarrollado en clínicas y hospitales ubicados en una capital del Nordeste de Brasil con 10 neurólogos que trabajan con personas con enfermedad de Alzheimer. La recolección de datos se llevó a cabo entre abril y mayo de 2012. Para la captura de datos empíricos, se utilizó un formulário, en entrevistas. El material fue tratado mediante la técnica de análisis de contenido temático. El análisis cualitativo reveló tres categorías: los objetivos de los cuidados paliativos; tipos de cuidados paliativos; y modos de afrontamiento. Los testimonios de los médicos que participaron de la investigación revelaron enfáticamente la importancia de los cuidados paliativos para pacientes de Alzheimer y su familia. Con base en el anterior, se pretende que esta investigación pueda seguir apoyando nuevos estudios en esa área.

Palabras Clave: Cuidados paliativos; enfermedad de Alzheimer; medicina; envejecimiento

\section{INTRODUÇÃO}

Os cuidados paliativos compreendem uma abordagem terapêutica que por meio da prevenção e do alívio do sofrimento, com a identificação precoce, a avaliação correta e o tratamento da dor e de outros problemas físicos, psicossociais e espirituais visam melhorar a qualidade de vida dos pacientes que enfrentam uma doença ameaçadora à vida, a qual afeta toda a dinâmica familiar ${ }^{1,2}$.

${ }^{\mathrm{I}}$ Médico. Doutorando do Programa de Pós-Graduação em Enfermagem da Universidade Federal da Paraíba. Pesquisador do Grupo Internacional de Estudos e Pesquisas sobre Envelhecimento e Representações Sociais. João Pessoa, Paraíba, Brasil. Email: qronaldo@ig.com.br.

"Enfermeira. Mestre e Pesquisadora do Núcleo de Estudos e Pesquisas em Bioética, Programa de Pós- Graduação de Enfermagem da Universidade Federal da Paraíba. João Pessoa, Paraíba, Brasil. E-mail: anazaccara@hotmail.com.

IIIMédica. Professora de Anestesiologia e Pesquisadora do Núcleo de Estudos e Pesquisas em Bioética da Universidade Federal da Paraíba. João Pessoa,

Paraíba, Brasil. E-mail: marciadmoreira@gmail.com.

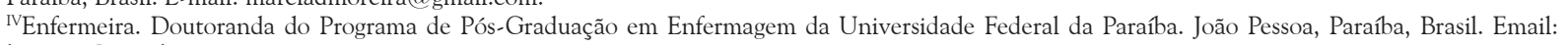
luipams@gmail.com

vEnfermeira. Doutora em Enfermagem. Professora do Programa de Pós-Graduação em Enfermagem da Universidade Federal da Paraíba. João Pessoa, Paraíba, Brasil. E-mail: solangefgc@gmail.com.

${ }^{\mathrm{V}}$ Enfermeira. Doutora em Enfermagem. Professora do Programa de Pós-Graduação em Enfermagem da Universidade Federal da Paraíba. João Pessoa,

Paraíba, Brasil. E-mail: alfaleda@hotmail.com 
Doenças que tendem a sugerir que a vida chegará ao fim geram diversos pensamentos e afetam o emocional tanto dos que possuem o diagnóstico da doença terminal e quanto daqueles que estão ao seu redor, podendo levar o individuo ao isolamento social e espacial, a consequência, geralmente, é a depressão e a solidão ${ }^{3}$. Esse distanciamento de pessoas amadas e queridas gera um sofrimento em um momento delicado e individual.

No tocante a pacientes portadores de Doença de Alzheimer (DA), o cuidado envolve questões éticas, com as quais o neurologista se depara frequentemente, que incluem o momento do diagnóstico, como e quando comunicá-lo, as consequências da progressão da doença e o seu manejo, a participação da família, o impacto do diagnóstico sobre ela e os cuidados com o paciente . $^{4}$

A diversidade de necessidades do portador de DA, na fase final de vida, e de sua família, associada à complexidade desse sofrimento, e nas suas abordagens específicas para cada doente, torna necessário o trabalho interdisciplinar em cuidados paliativos, cujos elementos são o médico, o enfermeiro, o psicólogo, o assistente social, o nutricionista e o assistente espiritual ${ }^{5-7}$.

Nesse sentido, o reconhecimento da importância da DA no cenário mundial, em particular, no Brasil, a dificuldade de se fornecer e receber o diagnóstico de acordo com a necessidade de promover uma qualidade de vida melhor para os pacientes, associado à implementação dos cuidados paliativos através dos profissionais que cuidam de pacientes acometidos por essa patologia, o que, certamente, contribuirá para a oferta de serviços com essa finalidade, foi o que motivou a realização desta pesquisa, do ponto de vista do profissional médico.

Diante das considerações apresentadas, este estudo teve o objetivo de apresentar concepções de neurologistas sobre a prática dos cuidados paliativos direcionada ao doente de Alzheimer.

\section{REVISÃo DE LITERATURA}

Para idosos com enfermidade crônica como a DA, a evolução para a morte sobrevém quando o paciente se encontra em uma condição de fragilidade, com declínio das funções biológicas e da qualidade de vida. Assim, é necessário promover uma assistência integral ao paciente que abranja, também, seus familiares, pois a aproximação da morte do ente querido desperta na família um desgaste físico e emocional ${ }^{5}$. Isso requer uma abordagem holística e humanizada que promova conforto e alivie os sintomas.

Assim, os cuidados paliativos se apresentam como uma ferramenta importante na prática assistencial para que o médico, integrante da equipe multidisciplinar, possa proporcionar uma assistência de qualidade no tratamento ao paciente crônico acometido pela DA com dignidade e respeito, ampliando a integralidade do atendimento ${ }^{6}$.
Nesse contexto, compete ao médico neurologista concluir o diagnóstico, entender os desejos da família, levar em consideração a posição e a circunstância desse ambiente familiar, o suporte de que o portador necessitará, quanto à terapêutica e aos cuidados de enfermagem, assim como um modo cuidar holístico, humanizado e singular de um paciente terminal de $\mathrm{DA}^{\mathrm{T}}$.

\section{Metodologia}

Trata-se de um estudo exploratório de abordagem qualitativa, desenvolvido em clínicas e hospitais localizados em uma capital do Nordeste brasileiro, com 10 médicos neurologistas, que trabalham com portadores de DA e que aceitaram participar da pesquisa voluntariamente.

Os dados foram coletados, mediante entrevista, no período de abril à maio de 2012. Para a apreensão do material empírico, utilizou-se um formulário contendo questões subjetivas, com vistas a atender ao objetivo, com questões subsidiadas no referencial dos cuidados paliativos e na prática profissional com pacientes acometidos por DA. A segunda parte contemplou as variáveis sexo, idade, tempo de formação acadêmica.

Vale ressaltar que a coleta foi feita após aprovação do projeto pelo Comitê de Ética em Pesquisa, do Hospital Universitário Lauro Wanderley, da Universidade Federal da Paraíba, sob o número do protocolo 184/10. Foi realizada depois que os médicos participantes do estudo assinaram o Termo de Consentimento Livre e Esclarecido, conforme estabelece a Resolução $\mathrm{n}^{\circ}$ 196/1996, do Conselho Nacional de Saúde, que dispõe sobre as normas e as diretrizes regulamentadoras de pesquisas com seres humanos em vigor no nosso país ${ }^{8}$.

Os dados coletados foram tratados por meio da técnica de análise de conteúdo temática, a qual é utilizada em pesquisas de campo da saúde, por abranger um conjunto de técnicas que favorecer o tramento dos significados dos discursos 9 . $O$ procedimento considerou as seguintes etapas: pré-análise, que compreendeu a leitura flutuante dos formulários respondidos, os quais constituiram o corpus; após a preparação do material houve a aplicação da técnica de análise de conteúdo e, posteriormente, houve a seleção das unidades de análise. Foram consideradas como unidades de contexto o parágrafo e como unidades de registro a frase. Para o recorte, a classificação e a categorização, optou-se pelo processo categorial indutivo, isto é, as categorias foram definidas a posteriori.

Em um segundo momento, após a exploração do material, quando foi aplicada ao corpus a técnica selecionada, houve o tratamento dos dados; a validação dos dados obtidos; as inferências e a interpretação dos resultados, com apoio do referencial teórico ${ }^{1-7,10-27}$. 


\section{Resultados}

Como categorização dos 10 médicos neurologistas participantes constatou-se que a faixa etária variou entre 42 e 74 anos, seis eram mulheres e quatro homens, dos quais nove possuiam mais de 20 anos de formação acadêmica. Vale ressaltar que apenas dois participantes cursaram uma pós-graduação strictu sensu.
Ao analisar os depoimentos dos médicos, emergiram três categorias: $1^{\text {a }}$ Categoria - Finalidades dos cuidados paliativos; 2a Categoria - Tipos de cuidado e suas respectivas subcategorias: biológico/físico, psicológico, medicamentoso e sociointeracional; $3^{\text {a }}$ Categoria - Formas de enfrentamento. As unidades de análises, que correspodem às frases coletadas, estão explicitadas na Figura 1.

\begin{tabular}{|c|c|c|}
\hline Categorias & Subcategorias & Unidades de análise \\
\hline $\begin{array}{l}\text { Finalidades dos } \\
\text { cuidados } \\
\text { paliativos }\end{array}$ & & $\begin{array}{l}{[\ldots] \text { não visa à cura do doente }[\ldots] \text { confortar-se em seu quadro clínico }} \\
{[\ldots] \text { alertando de ser patologia irreversível decadente }[. . .] \text { patologia }} \\
\text { comporta-se com vários níveis de gravidade }[. . .] \text { quadro de demência } \\
\text { em seu inicio }[\ldots] \text { doença crônica avançada ou em estágio terminal } \\
{[\ldots] \text { cuidados gerais que um paciente de doença grave necessita }[\ldots]} \\
\text { direcionado especialmente a fase grave ou avançada do paciente }[\ldots] \\
\text { ajudam a retardar o avanço da doença }[\ldots] \text { melhorar a condição de } \\
\text { vida do paciente }[\ldots] \text { necessita de outras pessoas que se tornam vitais } \\
\text { para o paciente }[\ldots] \text { cuidados mínimos necessários para aliviar o } \\
\text { sofrimento do paciente }[\ldots] \text { importantes na vida do paciente }[\ldots] \\
\text { melhorar sua condição de vida }[\ldots] \text { cuidados dados ao paciente e aos } \\
\text { cuidadores }[\ldots] \text { raro entre os familiares }[\ldots]\end{array}$ \\
\hline \multirow{4}{*}{$\begin{array}{l}2^{\mathrm{a}} \\
\text { Tipos de } \\
\text { cuidados } \\
\text { paliativos }\end{array}$} & Biológicos e físicos & $\begin{array}{l}\text { [...] minimizar os sintomas clínicos da doença [...] controle de } \\
\text { infecções e outras comorbidades [diabetes, hipertensão] [...] prevenção } \\
\text { das úlceras por pressão [...] mudança de decúbito e cuidados com } \\
\text { escaras [...] manutenção das suas atividades básicas [...] estímulo à } \\
\text { atividade física [...] cuidados físicos em relação à deambulação [...] } \\
\text { atividade física [...] cuidados direcionados às atividades da vida diária } \\
\text { e/ou intelectual [...] amenizar suas dores e suas dificuldades nas ações } \\
\text { do dia a dia [...] manutenção do suporte básico de vida [...] }\end{array}$ \\
\hline & Psicológicos & $\begin{array}{l}{[\ldots] \text { consciência da necessidade de lidar com a patologia [...] a família }} \\
\text { deve ficar ciente da patologia [...] conforto do paciente com doença } \\
\text { terminal [...] comunicação do diagnóstico de forma simples e suave } \\
{[\ldots] \text { controle de possíveis sintomas depressivos }[\ldots] \text { preservar a }} \\
\text { autoestima do paciente }[\ldots] \text { carinho e paciência }[\ldots] \text { incentivo às } \\
\text { atividades que estimulem as funções mentais }[\ldots] \text { (leituras, jogos, } \\
\text { palavras cruzadas) [...] instrução sobre a parte psicoemocional }[\ldots] \\
\text { psicoterapia [...] exercícios mentais como palavras cruzadas, leituras } \\
{[\ldots] \text { estimulação cognitiva }[\ldots] \text { psicoterapia }[\ldots] \text { terapia }} \\
\text { cognitivo/comportamental }[\ldots]\end{array}$ \\
\hline & medicamentoso & $\begin{array}{l}{[\ldots] \text { tratamento medicamentoso }[\ldots] \text { prescrevendo antipsicóticos e }} \\
\text { ansiolíticos }[\ldots] \text { antidepressivos }[\ldots] \text { usar vaso-ativadores tipo AAS e } \\
\text { outros medicamentos }[\ldots] \text { cuidados não medicamentosos }[\ldots]\end{array}$ \\
\hline & Sociointeracional & $\begin{array}{l}{[\ldots] \text { mantendo-o integrado ao máximo nas atividades regulares da }} \\
\text { família }[\ldots] \text { socialização }[\ldots] \text { grupos de apoio }[\ldots] \text { oficinas de memória } \\
{[\ldots] \text { atividades manuais: crochê, tricô }[\ldots] \text { inserção social }[\ldots]}\end{array}$ \\
\hline $\begin{array}{c}3^{\underline{a}} \\
\text { Formas de } \\
\text { enfrentamentos }\end{array}$ & & 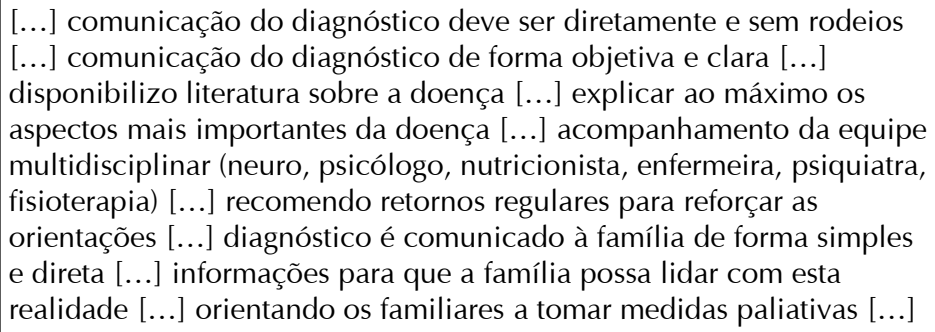 \\
\hline
\end{tabular}

FIGURA 1: Representação sinóptica das categorias e subcategorias segundo as unidades de análise. João Pessoa-PB, 2012 
$\mathrm{Na} 1^{\text {a }}$ categoria, a análise das falas demonstrou as finalidades dos cuidados paliativos, como certos relatos a seguir:

[...] não visa à cura do doente [...] confortar-se em seu quadro clínico [...] alertando de ser patologia irreversivel decadente [...] patologia comporta-se com vários níveis de gravidade [...]

A $2^{\text {a }}$ categoria deu origem às quatro subcategorias, as quais determinam falas características sobre os tipos de cuidados:

[...] controle de infecções e outras comorbidades (diabetes, hipertensão) [...] conforto do paciente com doença terminal [...] antidepressivos [...] inserção social [...]

Assim, a $3^{\text {a }}$ categoria emergiu de discursos como:

[...] explicar ao máximo os aspectos mais importantes da doença [...] acompanhamento da equipe multidisciplinar (neuro, psicólogo, nutricionista, enfermeira, psiquiatra, fisioterapia) [...]

\section{DiscUSSÃo}

O procedimento de análise adotado no estudo possibilitou a construção das três categorias já descritas.

\section{$1^{\text {a }}$ Categoria - Finalidades dos cuidados paliativos}

Os médicos neurologistas entendem os cuidados paliativos como uma ferramenta que visa aliviar o sofrimento do paciente com Alzheimer, oferecer conforto e melhorar sua condição de vida e a de seus familiares. Nessa perspectiva, seu foco não é mais a cura, ou seja, na atenção paliativa, os profissionais de saúde visam o cuidado e reconhecem o limite dos tratamentos e da medicina, assim como valorizam o conforto, o bem-estar, o zelo, a dedicação, a empatia e a atenção ao ser com doença terminal ${ }^{1,2,4,5}$.

Nesse sentido, merece destaque o conceito da Organização Mundial de Saúde, que considera os cuidados paliativos como uma abordagem que melhora a qualidade de vida de pacientes e de seus familiares, na presença de problemas associados a doenças que ameaçam a vida, mediante prevenção e alívio do sofrimento com a detecção precoce e o tratamento de dor ou outros problemas físicos, psicossociais e espirituais $^{10,11}$.

Os cuidados paliativos visam melhorar a qualidade de vida do paciente e de seus familiares, por meio de adequada avaliação e de tratamento para aliviar a dor e os sintomas, além de proporcionar suporte psicossocial e espiritual ${ }^{11-15}$. Para isso, é necessário promover uma assistência integral ao paciente, incluindo os seus familiares, uma vez que a aproximação da morte do ente querido provoca na família um desgaste físico e emociona ${ }^{14}$. Para isso, o fio condutor é uma abordagem holística e humanizada que promova conforto e alívio dos sintomas. Em outro enfoque, as falas dos médicos participantes do estudo demonstram a sensibilidade com o outro, a busca por seu conforto, o que culmina com um profundo respeito pelo paciente e por sua família e na valorização da dignidade de ambas as partes.

Estudo mostrou que importar-se com o outro e respeitar a vivência do momento de dor e de dificuldade possibilita a construção de uma relação de confiança entre os profissionais cuidadores e o paciente e seus familiares ${ }^{12}$.

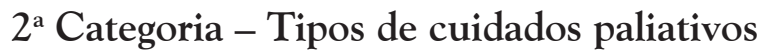

Apresenta quatro subcategorias apreendidas dos discursos dos médicos neurologistas participantes do estudo: cuidados biológicos/físicos; psicológicos; medicamentoso e sociointeracional.

\section{Subcategoria - Cuidados biológicos/físicos}

Diz respeito ao cuidado continuado e manejo de sintomas, controle, prevenção e manutenção das atividades básicas diárias e a crescente dependência gerada no decorrer do processo de evolução do paciente com Alzheimer.

Os cuidados paliativos preconizam que o atendimento ao paciente sem possibilidade terapêutica de cura deve ser pautado na escuta, na percepção, na compreensão e na identificação das necessidades para, só então, planejar ações. Vale ressaltar que tais cuidados emergem como uma estratégia por meio da qual se prestam cuidados levando-se em consideração a valorização da singularidade do paciente. Portanto, a equipe precisa estar disponível e receptiva para cuidar do paciente de Alzheimer em sua totalidade ${ }^{6}$.

Estudos demonstram que, quando os profissionais relatam a preocupação com a alimentação, inclusive, a possibilidade de indicar alimentação enteral, sugerem mais de um tipo de cuidados paliativos ${ }^{5-7,10-19}$. Conforme a American Dietetic Association, nesse tipo de cuidado, a alimentação deve propiciar prazer, conforto emocional, diminuição da ansiedade e aumento de autoestima, além de permitir maior integração e comunicação com seus familiares ${ }^{13}$.

Os cuidados paliativos visam evitar ou minimizar os sintomas clínicos da doença e controlar infecções e outras comorbidades como, por exemplo, diabetes, hipertensão, prevenção das úlceras por pressão e mudança de decúbito e cuidados com escaras, alívio da dor, entre outros ${ }^{5-710-27}$.

\section{Subcategoria - Cuidados psicológicos}

Evidencia a preocupação dos neurologistas com a integridade, por maior tempo possível, da estrutura psicológica do paciente com DA e daqueles envolvidos em seu cuidado. Logo, humanizar o atendimento às necessidades de todos os que lidam com a doença 
sem possibilidades de cura, melhorando a qualidade de vida, só é possível com cuidados paliativos, em seu sentido primeiro de amenizar o transtorno do processo desde o momento do diagnóstico ${ }^{14}$.

Do ponto de vista psicológico, oferecer cuidados paliativos implica atender aos pacientes com Alzheimer, aos seus cuidadores familiares e aos profissionais, na perspectiva de cinco dimensões principais: física, psicológica propriamente dita, social, espiritual e ética, desde o momento do diagnóstico, visando manter ou melhorar a qualidade de vida de todos os envolvidos ${ }^{15}$. Para tanto, não basta controlar e aliviar os sintomas, mas também dar apoio para manter a estrutura familiar e promover a comunicação entre os membros da unidade de cuidados, amparo emocional e social.

Diante de um diagnóstico de uma doença que, inexoravelmente, é ameaçadora e sem possibilidade de cura, todos se tornam vulneráveis e são afetados de alguma forma. Por isso, cabe ao psicólogo atuar como ponto de ligação entre a família e a equipe de cuidados paliativos, visando buscar respostas para enfrentar a fase final de vida, e contribuir de maneira a fortalecer a família, para que se comunique bem com a equipe, desenvolva seu senso de controle sobre o que sabe ou o que não sabe, entenda o processo da morte e identifique sintomas que requerem atenção imediata, para ser um agente eficiente nesse processo ${ }^{15}$.

Os sintomas psicológicos e comportamentais das demências podem ser tratados com terapia comportamental, estimulação multissensorial, estimulação cognitiva, exercícios físicos, musicoterapia, recreação, arteterapia, estímulo à expressão criativa e programas de contação de histórias que estimulam a imaginação ${ }^{16}$. Tais atividades podem trazer benefícios relacionados ao humor, estimular a memória e levar os pacientes a compartilharem suas experiências e valores.

\section{Subcategoria - Cuidado medicamentoso}

Nas descrições dos profissionais, há nítida preocupação com cuidados paliativos no conhecimento sobre as medicações que deverão ser usadas em pacientes com doença sem possibilidade de cura como a DA.

De acordo com estudos sobre concepções dos cuidados paliativos, a dor ocorre em indivíduos que vivenciam uma série de desconfortos de caráter físico, psíquico, social e espiritual, como lesões cutâneas, odores desagradáveis, anorexia, caquexia, insônia, fadiga, luto antecipado, dificuldades econômicas, depressão, entre outros ${ }^{1-3,7,10-27}$.

Sabe-se que, na prática, uma dor mal controlada causa impacto, além do âmbito físico, tanto para o indivíduo doente quanto para a família e os profissionais de saúde. Daí nasceu o termo dor total, uma vez que a dor, nos seres humanos, é um quadro que, além da nocicepção (quantidade de tecido lesado), envolve fatores físicos, emocionais, sociais e espirituais, que influenciam na vivência e na expressão da queixa de dor. Desse modo, o controle e o alívio da dor e dos demais sintomas são um direito do indivíduo e um dever dos profissionais, que devem criar estratégias para diminuir o sofrimento provocado por esse quadro ${ }^{17}$.

Vale destacar que estudos sugerem que antipsicóticos como risperidona ( $1 \mathrm{mg} / \mathrm{dia})$ e olanzapina (5 a $10 \mathrm{mg} /$ dia) são efetivos como terapia medicamentosa a pacientes com Alzheimer. Entretanto, nos últimos anos, autoridades reguladoras, inclusive a Agência Nacional de Vigilância Sanitária (ANVISA), notificaram que ambas as substâncias aumentam o risco de eventos cerebrovasculares ${ }^{18}$.

\section{Subcategoria - Cuidados sociointeracionais}

Identifica-se a necessidade de manter a socialização do paciente e seu engajamento em atividades produtivas, na tentativa de preservar e aproveitar suas reservas cognitivas para melhorar sua qualidade de vida e de seus familiares. Diante dos depoimentos dos médicos participantes do estudo, no que concerne à atenção ao aspecto social do paciente, destaca-se a inserção do familiar durante todo o processo de tratamento. Tal atitude é fundamental para os cuidados requeridos pelo paciente, e isso confirma a necessidade de se escutar seu sofrimento e disposição para o aprendizado para lidar com as dificuldades que surgem no decorrer da doença. Assim, o estímulo ao convívio familiar e o fortalecimento dos laços em família têm como objetivo principal reduzir a ansiedade e o sofrimento ${ }^{19}$.

Autores têm apontado melhora no desempenho cognitivo e funcional de pacientes em fases iniciais da DA, com a utilização de intervenções psicossociais, principalmente quando associado ao tratamento farmacológico preconizado. Contudo, poucos estudos se propuseram a elucidar a eficácia do tratamento não medicamentoso de sujeitos em fases mais evoluídas da doença ${ }^{20}$.

Quanto mais cedo for feito o diagnóstico de DA e se iniciar o tratamento, incluindo medidas sociointerativas - reabilitação neuropsicológica - melhores resultados serão obtidos, pois o paciente poderá manter-se em um nível cognitivo e funcional mais elevado. Isso possibilitará, ainda, que ele permaneça mais tempo independente e se evitem sobrecargas para seus familiares. Pequenas alterações cognitivas ou até mesmo a estabilização de algumas funções cognitivas já revelam um grande ganho, no caso de pacientes com doença neurodegenerativa.

\section{$3^{\text {a }}$ Categoria - Formas de enfrentamento}

Ressalta as maneiras de se lidar com a doença, o que abrange desde os familiares, quando são comunicados de forma a terem as informações necessárias, bem como os próprios doentes, que passam a ter um acompanhamento interdisciplinar em que há uma interação 
entre: médico, enfermeiro, psicólogo, nutricionista, pacientes e familiares. Tais aspectos são evidentes nos depoimentos dos profissionais pesquisados sobre a troca de informações entre médico e familiares do portador de Alzheimer, em que valorizam essa relação.

Os discursos dos participantes expressam que eles entendem a importância de a informação do diagnóstico ser dada à família. Nessa perspectiva, os membros da família assumem um papel de destaque e devem ser vistos como responsáveis pela saúde dos seus, razão pela qual devem ser ouvidos, valorizados e estimulados a participar de todas as fases da doença, a partir do seu diagnóstico e durante todo o processo do cuidar. Isso é relevante, pois a DA, por suas características, representa, indubitavelmente, um pesadelo na vida de seu portador e de seus convivas ${ }^{21}$.

No que concerne à comunicação do diagnóstico aos familiares de paciente com uma doença crônica, degenerativa e progressiva, como Alzheimer, é possível salientar que está intrinsecamente ligada à experiência prévia pessoal e profissional dos informantes. Além disso, é preciso saber se a notícia deve ser dada aos familiares ou ao próprio paciente ${ }^{22}$. Na patologia em questão, salvo casos excepcionais, a troca de informações, geralmente, é mantida com a família, visto que o indivíduo se encontra sem condições de receber seu diagnóstico pelo comprometimento neurológico que pode estar instalado em graus variados.

Assim como o diagnóstico, as orientações sobre os cuidados que devem ser dispensados ao paciente são referidas à família. Compreende-se a dimensão da importância da família no contexto em discussão. Um estudo verificou que $70 \%$ dos médicos pesquisados afirmam dar apoio e estabelecer uma relação de confiança com os familiares do paciente, demonstrando a consideração dispensada pelos profissionais aos convivas do doente ${ }^{23}$. Todavia, para o enfrentamento da doença, é necessário falar a verdade, porquanto ela é vital, e há formas adequadas de transmiti-la. A maneira como o médico interage com os familiares e os pacientes são de extrema importância, por ser o início de um processo doloroso, sofrido e difícil para todos os envolvidos. Provavelmente, esse momento poderá ser decisivo para a condução da tempestade de sentimentos e atitudes que virão à tona e direcionarão o binômio saúde e doença, que envolverá o paciente e seus familiares ${ }^{24}$.

O estudo adverte, todavia, que a atitude do profissional tanto pode tranquilizar o indivíduo, frente às mudanças em sua vida, quanto piorar o estado de saúde emocional. Portanto, a forma de lidar com o diagnóstico deve ser bem trabalhada, visto que a maneira como essa tarefa é realizada guiará os sentimentos e as atitudes que conduzirão pacientes e familiares ao enfrentamento da doençą ${ }^{23}$.

Sob esse prisma, trata-se da dificuldade de comunicar o diagnóstico de uma doença incurável, tendo em vista que essa tarefa deve ser realizada de forma processual e cautelosa, a partir do momento que suscita diversas reações e fantasias, culminando com o medo da morte ${ }^{13}$.

Nesse sentido, estudo destaca as estratégias utilizadas para promover a boa comunicação, quais sejam: a utilização de comunicação verbal e não verbal para subsidiar o cuidar humanizado ao paciente, de forma a proporcionar apoio, segurança, confiança, transmitir força e esperança, o que é primordial no contexto dos cuidados paliativos ${ }^{25}$.

Além das estratégias de comunicação, a avaliação e o manejo de sintomas comportamentais e psicológicos da DA são um desafio, e dilemas éticos surgem frequentemente. Pesquisa aponta que os médicos, muitas vezes, não percebem uma desconexão entre diretrizes baseadas em evidências e os desafios do tratamento da DA. Tendo em vista o fato de ser uma doença progressiva, o estudo propõe uma abordagem de cuidados paliativos que priorize o reconhecimento da pessoalidade e da preservação da dignidade ${ }^{26}$.

Principalmente, no momento em que os pacientes com DA atingem a fase final da doença, o que reduz sua capacidade para fornecer queixas subjetivas pertinentes a dor e desconfortos. Dessa forma, explorar o processo de adoecimento, as questões éticas e considerações clínicas importantes são necessárias para melhorar a qualidade de atendimento para pacientes em estágio final da $\mathrm{DA}^{27}$.

\section{Conclusão}

Nesta pesquisa, ficou evidente a importância dessa modalidade de cuidado, oferecer suporte para suprir as necessidades biológicas, psicológicas e sociais do paciente e de se dar mais atenção à terapia medicamentosa e às formas de se enfrentar a doença, a partir das concepções de médicos neurologistas sobre os cuidados paliativos, na prática profissional, frente ao doente de Alzheimer.

Verificou-se posição unânime diante da DA - a família se reveste de importância fundamental, visto que é ela quem recebe o diagnóstico, portanto, é responsável pelo paciente durante todo o processo do adoecimento. Os profissionais ressaltaram também a importância da comunicação como uma estratégia fundamental na prática dos cuidados paliativos.

Os depoimentos dos médicos participantes da investigação deixam transparecer, de modo enfático, a relevância dos cuidados paliativos para o doente de Alzheimer e sua família. Ante o exposto, pretende-se que esta pesquisa possa subsidiar novos estudos nessa área.

A limitação encontrada na realização deste estudo foi a inexistência de uma unidade específica para este tipo de abordagem terapêutica; percebe-se, na literatura abordada, que essa problemática brasileira requer dedicação dos profissionais e investimento em capacitação profissional na área de cuidados paliativos e pacientes terminais. 


\section{REFERÊNCIAS}

1.Rodrigues IG, Zago MMF. A morte e o morrer: maior desafio de uma equipe de cuidados paliativos. Cienc Cuid Saude. 2012; 11: 31-8.

2.Rodrigues IG, Zago MMF. Cuidados paliativos: realidade ou utopia? Cienc Cuid Saude. 2009; 8: 136-41. 3.Vasques TCS, Lunardi VL, Silveira RS, Gomes GC, Lunardi Filho WD, Pintanel AC. Percepção dos trabalhadores de enfermagem acerca da implementação dos cuidados paliativos. Rev enferm UERJ. 2013; 21: 16-22. 4.Paula JJ, Bertola L, Nicolato R, Moraes EN, MalloyDiniz LF. Evaluating language comprehension in $\mathrm{Alz}$ heimer's disease: the use of the Token Test. Arq NeuroPsiquiatr. 2012; 70: 435-40.

5.Bifulco VA, Iochida LC. A formação na graduação dos profissionais de saúde e a educação para o cuidado de pacientes fora de recursos terapêuticos de cura. Rev bras educ med. 2009; 33(1): 92-100.

6.Machado J, Caram CLB, Frank AA, Soares EA, Laks J. Estado nutricional na doença de Alzheimer. Rev Assoc Med Bras. 2009; 55: 188-91.

7.Blasco PG. O médico perante a morte. Rev Bras de Cuidados Paliativos. 2009; 2(4): 7-13.

8.Ministério da Saúde (Br). Conselho Nacional de Saúde, Comissão Nacional de Ética em Pesquisa. Resolução n⿳o 196 de 10 de outubro de 1996: diretrizes e normas regulamentadoras de pesquisa envolvendo seres humanos. Brasília (DF): CNS; 1996.

9.Bardin L. Análise de conteúdo. Lisboa (Pt): Edições 70; 2012.

10.Mencarelli VL, Bastidas LS, Aiello Vaisberg TMJA. A difícil notícia da síndrome de imunodeficiência adquirida para jovens: considerações psicanalíticas com base na perspectiva winnicottiana. Psicologia: teoria e prática. 2008; $10(2): 106-20$.

11. World Health Organization (WHO). Who definition of palliative care [Internet]. 2012. [citado em 18 fev 2014]. Disponível em: http://www.who.int/cancer/ palliative/definition/en/.

12.Floriani CA, Schramm FR. Cuidados paliativos: interfaces, conflitos e necessidades. Ciênc Saude Colet. 2008; 13: 2123-32.

13.Benarroz MO, Faillace GBD, Barbosa LA. Bioética e nutrição em cuidados paliativos oncológicos em adultos.
Cad Saude Publica. 2009; 25: 1875-82.

14.Santos FS. Cuidados paliativos: diretrizes, humanização e alívio de sintomas. São Paulo: Editora Atheneu; 2011. 15.Melo AC, Valero FF, Menezes M. A intervenção psicológica em cuidados paliativos. Psic., Saúde \& Doenças (Lisboa). 2013; 14: 452-69.

16.Phillips L, Reid-Arndt S, Pak Y. Effects of a creative expression intervention on emotions, communication, and quality of life in persons with dementia. Nurs Res. 2010; 59: 417-25.

17.Silva EP, Sudigursky D. Concepções sobre cuidados paliativos: revisão bibliográfica. Acta Paul Enferm. 2008; 21: $504-8$.

18.Caixeta L. Doença de Alzheimer. Porto Alegre (RS): Artmed; 2012.

19.Nunes MGS, Rodrigues BMRD. Tratamento paliativo: perspectiva da família. Rev enferm UERJ. 2012; 20:338-43. 20.Cunha FCM, Cunha LCM, Silva HM, Couto EAB. Abordagem funcional e centrada no cliente na reabilitação de idoso com Demência de Alzheimer avançada: relato de caso. Rev Ter Ocup Univ São Paulo. 2011; 22:145-52. 21.Neumann S, Dias C. Living with Alzheimer's Disease in the family. Journal of Nursing UFPE on line. 2011; 5: 967:73. 22. Oliveira FT, Aparecida D, Marengo FMO, Silva RHA. Bioética e humanização na fase final da vida: visão de médicos. Rev Bioética. 2011; 19: 247-58.

23.Peternella FMN, Marcon SS. Descobrindo a Doença de Parkinson: impacto para o parkinsoniano e seu familiar. Rev Bras Enferm. 2009; 62: 25-31.

24.Araujo MMT, Silva MJP. O conhecimento de estratégias de comunicação no atendimento à dimensão emocional em cuidados paliativos. Texto contexto - enferm. 2012; 21: 121-9.

25.Brito FM, Costa ICP, Costa SFG, Andrade CG, Santos KFO, Francisco DP. Comunicação na iminência da morte: percepções e estratégia adotada para humanizar o cuidar em enfermagem. Esc Anna Nery. 2014; 18: 317-22 26.Passmore MJ1, Ho A, Gallagher R. Behavioral and psychological symptoms in moderate to severe Alzheimer's disease: a palliative care approach emphasizing recognition of personhood and preservation of dignity. J Alzheimers Dis. 2012; 29: 1-13.

27. Yeaman PA, Ford JL, Kim KY. Providing quality palliative care in end-stage Alzheimer disease. Am J Hosp Palliat Care. 2013; 30: 499-502. 